\section{Wieland, Heinrich Otto}

\section{W. Hubl}

Ehem. Krankenhaus Dresden-Friedrichstadt, Institut für Klinische Chemie und Labormedizin, Dresden, Deutschland

Lebensdaten Deutscher Chemiker, geboren am 4. Juni 1877 in Pforzheim, gestorben am 5. August 1957 in München. Wieland studierte ab 1896 in München, Berlin und Stuttgart Chemie und promovierte im Jahr 1901 bei Johannes Thiele. Im Jahr 1904 habilitierte er zum Thema Addition von $\mathrm{N}_{2} \mathrm{O}_{3}$ an Doppelbindungen. 1908 heiratete er Josephine Bartmann, sie bekommen drei Söhne und eine Tochter. Im Jahr 1917 übernahm er die Leitung der Abteilung für Kampfstoffsynthese an Fritz Habers (1868-1934) Kaiser-Wilhelm-Institut für physikalische Chemie und Elektrochemie in BerlinDahlem. Dort entwickelte er Kampfstoffe, z. B. Lost. In dieser Zeit pendelte er gleichzeitig zur TH München. 1921 erhielt er eine Professur an der Albert-Ludwigs-Universität in Freiburg. Im Jahr 1925 erhielt er den Ruf an die Technische Universität München als Nachfolger von Richard Willstätter (1872-1942). Arbeitsschwerpunkte waren Untersuchungen zu Cholsäure, > Cholesterin und einem Krötengift. Es gelang ihm, die Struktur der Steroide aufzuklären. Seine Forschungen, wie auch die zum indianischen Pfeilgift, galten im Dritten Reich als kriegswichtig, wodurch er in der Lage war, zahlreiche so genannte „Halbjuden“ als „Gäste des Geheimrats" aufzunehmen und zur Entlastung von Hans Conrad Leipelt (1921-1945) vor Gericht auszusagen.
Verdienste Ab 1912 begann Wieland in enger Zusammenarbeit mit Windaus ( $\triangleright$ Windaus, Adolf Otto Reinhold) die Untersuchungen zur Strukturaufklärung mittels oxidativer Ringöffnung, die später zur Konstitutionsermittlung der - Gallensäuren diente. Für diese Arbeit erhielt er 1927 den Nobelpreis für Chemie zu den Forschungen über die Zusammensetzung der Gallensäure und verwandter Substanzen. Er war Ritter des Ordens Pour le merite und Träger des Bundesverdienstkreuzes. 1925 wurde er zum Mitglied der Deutschen Akademie der Naturforscher Leopoldina gewählt. 1929 wurde er in die American Academy of Arts and Sciences sowie als korrespondierendes Mitglied in die damalige Sowjetische Akademie der Wissenschaften aufgenommen. Seit 1964 wird der Heinrich-Wieland-Preis vergeben für innovative wissenschaftliche Arbeiten in den Bereichen Biochemie, Chemie, Physiologie und klinische Medizin der Fette und Lipide, heute für biologisch aktive Moleküle und Systeme sowie deren klinische Bedeutung in der Chemie, Biochemie und Physiologie.

\section{Literatur}

Nobel Lectures (1966) Chemistry 1922-1941. Elsevier Publishing Company, Amsterdam

Witkop B (1977) Heinrich Wieland hundert Jahre. Sein Werk und Vermächtnis heute. Angewandte Chemie 89:575-589 